\title{
Engineering optimization with the fast boundary element method
}

\author{
I. Ostanin, A. Mikhalev, D. Zorin \& I. Oseledets \\ Skolkovo Institute of Science and Technology, Russia
}

\begin{abstract}
A wide variety of engineering design tasks can be formulated as optimization problems where the shape and topology of an elastic domain are optimized to reduce costs, e.g. global compliance, while satisfying certain constraints, such as volume constraint. We propose an application of a fast 3D boundary element code to the problems of shape and topology optimization. Our algorithm is based on the formalism of topological derivatives. Adaptive tree strategy of sampling of topological derivatives inside the domain, high performance algebraic solver and the analysis of optimization problem in reduced dimensions promise state of the art performance in the problems of engineering optimization. The approach can be applied to various optimization problems, such as minimization of compliance of an elastic structure or minimization of the distance from a current homogenized elasticity tensor of a periodic structure to the desired one. The efficiency of the approach is illustrated with a numerical example.
\end{abstract}

\section{Introduction}

Structural, shape and topology optimization are classical areas of modern engineering science. Pioneer works on structural optimization emerged in the early sixties [1]. A number of efficient shape optimization techniques have been developed subsequently, including boundary variation algorithms [2], level set methods [3], homogenization [4]. A number of works illustrated that boundary variation optimization approaches are naturally treated with boundary element method (BEM) [5]. Few recent works [6, 7] have also demonstrated that BEM can be a convenient tool for topological-shape optimization. Significant work has been done in the adjacent field of shape sensitivity analysis in elastodynamics using fast multipole BEM [8]. Modern fast BEM techniques (e.g. [9, 10]) enable the 
analysis of the systems with tens of millions degrees of freedom and can make shape optimization procedures a lot more efficient than the intellectual labour of an experienced engineer.

On the other hand, the emergence and rapid development of additive technologies $[11,12]$ eliminated existing technological limitations on admissible design shapes, and led to the situation when any optimized shape can be immediately produced by the 3D printing device. These factors revived the interest in large-scale shape and topology optimization techniques.

In this work we present our preliminary results on such a technique which is based on the boundary element method (BEM) [13], topological derivatives $[14,15]$ and the fast algebraic solver that utilize implicit $\mathcal{H}^{2}$ representation of the system matrix [16]. Other key points of the proposed method should include a fast algorithm of calculation of fields inside the domain and hierarchical tree sampling of these fields. This combination promises to reach state of the art performance in topological-shape optimization.

\section{Mathematical foundations of the method}

Our optimization procedure is based on the direct formulation of BEM for elasticity [13]. The basic integral equation formulation uses Somigliana displacement identity and in the absence of body forces for the domain $\Omega$ with a smooth enough boundary $\Gamma$ it can be written as:

$$
\frac{1}{2} u_{i}(\xi)=\int_{\Gamma} U_{i j}(\xi, x) p_{j} d \Gamma-\int_{\Gamma} P_{i j}(\xi, x) u_{j} d \Gamma
$$

where $u_{i}(x)\left(p_{i}(x)\right)$ is the displacement (traction) at the point on a boundary of the domain, $U_{i j}(x, \xi)\left(P_{i j}(x, \xi)\right)$ are the corresponding fundamental solutions. For the case of a linear isotropic elastic material with the shear modulus $G$ and Poisson's ratio $\nu$ they are given by:

$$
\begin{gathered}
U_{i j}(\xi, x)=\frac{1}{16 \pi(1-\nu) G r}\left((3-4 \nu) \delta_{i j}+r_{, i} r_{, j}\right) \\
P_{i j}(\xi, x)=\frac{1}{8 \pi(1-\nu) r^{2}} \\
\times\left[\frac{\partial r}{\partial n}\left((1-2 \nu) \delta_{i j}+3 r_{, i} r_{, j}\right)-(1-2 v)\left(r_{, i} n_{j}-r_{, j} n_{i}\right)\right]
\end{gathered}
$$

where $r=|\xi-x|$. Once the solution on the boundary is found, the stress at the point inside the domain can be calculated according to:

$$
\sigma_{i j}(p)=-\int_{\Gamma} u_{k}(x) S_{k i j}(p, x) d \Gamma+\int_{\Gamma} t_{k}(x) D_{k i j}(p, x) d \Gamma
$$

where $D_{k i j}(p, x)$ and $S_{k i j}(p, x)$ are given by: 


$$
\begin{gathered}
D_{k i j}(p, x)=\frac{1-2 \nu}{2 \pi(1-\nu) r^{2}}\left(\delta_{k i} r_{, j}+\delta_{k j} r_{, i}-\delta_{i j} r_{, k}+\frac{3}{1-2 \nu} r_{, i} r_{, j} r_{, k}\right) \\
S_{k i j}(p, x)=\frac{3-6 \nu}{4 \pi(1-\nu) r^{3}} \\
\times\left[\delta_{i j} r_{, k}+\frac{\nu}{1-2 \nu}\left(\delta_{k i} r_{, j}+\delta_{k j} r_{, i}\right)-\frac{5}{1-2 \nu} r_{, i} r_{, j} r_{, k}\right] \frac{\partial r}{\partial n} \\
+\frac{1-2 \nu}{4 \pi(1-\nu) r^{3}} \\
\times\left[\frac{3 \nu}{1-2 \nu}\left(n_{i} r_{, j} r_{, k}+n_{j} r_{, i} r_{, k}\right)+3 n_{k} r_{, i} r_{, j}+n_{j} \delta_{k i}+n_{i} \delta_{k j}-\frac{1-4 \nu}{1-2 \nu} n_{k} \delta_{i j}\right]
\end{gathered}
$$

The numerical treatment of this formulation is presented in the next section. The optimization procedure utilizes the concept of topological derivative (TD) - a cost of making an infinitesimal spherical cavity with a center in a given point of the domain. For the case of strain energy (compliance) cost functional and 3D linear isotropic elasticity, the analytical expression of TD is available [14]:

$$
D^{T}(p)=\frac{3}{4 E} \frac{1-\nu}{7-5 \nu}\left[10(1+\nu) \sigma(p) \cdot \sigma(p)-(1+5 \nu) \operatorname{tr} \sigma(p)^{2}\right]
$$

where $E$ is the material's Young's modulus. It is important to note, that our approach is not limited to the particular functional, and can be used to optimize other functionals. One of the most promising applications is the optimization of the elastic tensor of a periodic cell $[17,18]$. Optimization strategies employing TDs are usually based on the progressive elimination of the material. One particular method of the optimization of a voxelized structure is presented in the example section.

\section{Numerical solution}

The key feature for the large scale optimization procedure is the complexity growth for increasing number of degrees of freedom. All the approaches that use domain discretization cannot in principle be more efficient than $O(M)$, where $M$ is the number of volume elements (finite elements, finite volumes etc.). Since boundary element techniques require only the discretization of the boundary, fast solvers (e.g. [9, 10]) allow finding the boundary solution for $O(N)$ or $O(N \cdot \log (N))$ operations $\left(N \sim M^{2 / 3}\right)$. The TD computation is then a volumetric computation and if done in a naive way, requires at least $\mathcal{O}(N)$ operations. Moreover, even the computation and storage of the BEM matrix (which is dense) requires $N^{2}$ operations. There are different approaches, that can be traced back to the well-known fast multipole method, that reduce the complexity of 
the matrix-by-vector product to $\mathcal{O}\left(N \log ^{\alpha} N \log ^{\beta} \varepsilon^{-1}\right.$, where $\varepsilon$ is the accuracy. The derivation of analytical multipole-type expansion for the elasticity problem is possible, but very technical. Instead, one can use black-box type methods that are kernel-independent [19-21]. In our work we use the h2tools software package (http://bitbucket.org/muxas/h2tools) [16], in order to set up a fast iterative solver of the boundary integral equation (1). The method requires only the information about the geometry of the problem and a function that evaluates prescribed element of the matrix.

Using the method of collocations and piecewise-constant approximation [13], one can discretize the equation (1) into the following system of linear equations:

$$
\left(\frac{1}{2} I+\Delta P\right) \cdot u=\Delta U \cdot p
$$

where $\Delta P_{i j}=\int_{S_{k}} P_{m n}\left(\xi_{l}, x\right) d S_{k}, \Delta U_{i j}=\int_{S_{k}} U_{m n}\left(\xi_{l}, x\right) d S_{k}, i=3 k+m$, $j=3 l+n, \xi_{l}$ is $l$-th collocation point and $S_{k}$ is the area of $k$-th boundary element. Regular terms $(k \neq l)$ are evaluated numerically, singular terms $(k=l)$ are evaluated analytically (see [13] for details).

After straightforward rearrangement of columns in 8 , we obtain the following system of linear equations, where all unknowns appear in vector $x$, whereas tractions or displacements known from boundary conditions appear in vector $y$.

$$
A \cdot x=B \cdot y
$$

Within our approach, equation (9) is solved using $\mathcal{H}^{2}$ factorization of matrices $A$ and $B$, without direct calculation of all elements in these matrices. Our method to compute $\mathcal{H}^{2}$ factorization uses recently developed multicharge Barnes-Hut method (MCBH) [16], which provides $O(N)$ performance (up to logarithmic factor) in factorization and calculation of matrix-vector products needed for fast evaluation of the right-hand side of 9 , as well as for setting up an iterative solver. This allows the analysis of large-scale problems.

Our method for topology optimization process requires TD computations inside the domain using 4,5,2,7. Straightforward evaluation of a TD at a point inside the domain takes $O(N)$ operations. Application of fast methods (for example, MCBH method adapted for a single-point evaluation) can reduce this $\operatorname{cost}$ to $O(\log N)$. In combination with adaptive tree sampling of TDs, as described in [22], this approach can boil the total complexity of this step down to $O(N \log N)$.

\section{Example}

In this section we discuss a simple benchmark example that allows us to assess the capabilities of our approach. Consider a 2D problem of optimization of a shape and topology of a fixed support, previously discussed in [22]. The initial domain is a square (Fig. 1(a)). The left side of the support is rigidly fixed, and the point load is applied at the upper-right angle. 


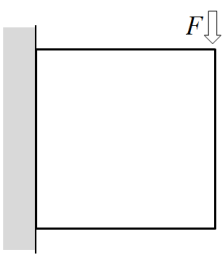

(a)

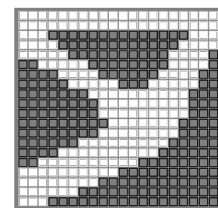

(b)

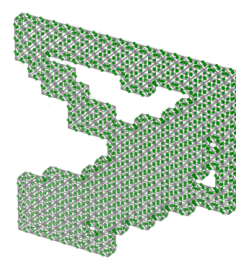

(c)

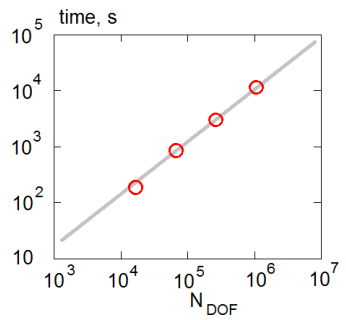

(d)

Figure 1: (a) Initial problem setup (b) 2D solution, as obtained in [22]; (c) 3D solution obtained with our code (d) Time per $\mathcal{H}^{2}$ matrix factorizations as a function of number of problem's degrees of freedom $N_{d o f}$.

Our algorithm of topology optimization is based on successive elimination of voxelized structure, which is a straightforward generalization of the algorithm presented in [22] - at every iteration the material is eliminated if the value of TD evaluated at the center of the voxel according to 7 is less than the certain critical level, which is defined as $D_{c}=D_{\min }+C \cdot\left(D_{\max }-D_{\min }\right), D_{\max }$ and $D_{\min }$ being maximum and minimum values of the TD, and $C$ is the constant (chosen in range between 0.001 and 0.05 ). Figures $1(\mathrm{~b})$ and 1(c) give the comparison of the 2D plane stress solution obtained in [22] and 3D solution obtained with 3D fast BEM. The results are in reasonable agreement.

Figure 1(d) shows the time for the computation of $\mathcal{H}^{2}$-factorizations of matrices $A$ and $B$ (the most computationally expensive part of the solver) as a function of the number of degrees of freedom in the model $\left(N_{d o f}\right)$. Calculations were performed on a regular laptop computer. It appears that our solver is reasonably scalable and can be used for optimization problems with millions of degrees of freedom (which correspond to billion "virtual" degrees of freedom if the volumetric mesh was used).

\section{Conclusions}

In this note we presented our recent results on application of fast 3D BEM techniques to the problems of topological-shape optimization. The method seems to be very promising for the mechanical problems of large-scale topology optimization. Immediate future work includes the development of fast algorithm of calculations of fields inside the domain, as well as the algorithm of tree sampling of these fields that has shown its efficiency in 2D [22].

\section{Acknowledgement}

The authors gratefully acknowledge the financial support from Russian National Foundation under the grant 15-11-00033. 


\section{References}

[1] Schmit, L.A., Structural design by systematic synthesis. Proceedings, 2nd Conference on Electronic Computation, ASCE, New York, 1960.

[2] Azegami, H., Shimoda, M., Katamine, E. \& Wu, Z., A domain optimization technique for elliptic boundary value problems. Computer Aided Optimization Design of Structures IV, Structural Optimization, eds. S. Hernandez, M. El-Sayed \& C. Brebbia, Computational Mechanics Publications, Southampton, 1995.

[3] Allaire, G., Jouve, F. \& Toader, A.M., Structural optimization using sensitivity analysis and a level-set method. Journal of Computational Physics, 194(1), pp. 363-393, 2004.

[4] Allaire, G., Bonnetier, E., Francfort, G. \& Jouve, F., Shape optimization by the homogenization method. Numerische Mathematik, pp. 27-68, 1997.

[5] Meric, R.A. \& Saigal, S., Load sensitivity analyses of elastic structures by differential and boundary integral equation formulations. Structural Optimization, 3, pp. 240-246, 1992.

[6] Marczak, R., Optimization of elastic structures using boundary element and a topological-shape sensitivity formulation. Mechanics of Solids in Brazil, Brasilian Society of Mechanical Sciences and Engineering, pp. 279-293, 2007.

[7] Bertsch, C., Cisilino, A., Langer, S. \& Reese, S., Topology optimization of 3d elastic structures using boundary elements. Proc. Appl. Math. Mech. 8, pp. 10771-10772, 2008.

[8] Nemitz, N. \& Bonnet, M., Topological sensitivity and fmm-accelerated bem applied to 3d acoustic inverse scattering. Engineering Analysis with Boundary Elements, 32, pp. 957-970, 2008.

[9] Nishimura, N., Fast multipole accelerated boundary integral equation methods. Applied Mechanics Reviews, 55(4), pp. 299-324, 2002.

[10] Benedetti, I., Aliabadi, M. \& Davi, G., A fast 3d dual boundary element method based on hierarchical matrices. International Journal of Solids and Structures, 45, pp. 2355-2376, 2008.

[11] Melchels, F.P.W., Feijen, J. \& Grijpma, D.W., A review on stereolithography and its applications in biomedical engineering. Biomaterials, 31(24), pp. 6121-6130, 2010.

[12] Gross, B., Erkal, J., Lockwood, S., Chen, C. \& Spence, D., An evaluation of $3 \mathrm{~d}$ printing and its potential impact on biotechnology and the chemical sciences. Analytical Chemistry, 86(7), pp. 3240-3253, 2014.

[13] Cruse, T.A., Numerical solutions in three-dimensional elastostatics. Int $J$ Solids Structures, 5, pp. 1259-1274, 1969.

[14] Novotny, A., Feijoo, R., Taroco, E. \& Padra, C., Topological sensitivity analysis for three-dimensional linear elasticity problem. Computational Methods in Applied Mechanics and Engineering, (196), pp. 4354-4364, 2007. 
[15] Sokolovski, J. \& Zochovski, A., Topological derivatives of shape functionals for elasticity systems. ISNM International Series of Numerical Mathematics, 139, pp. 231-244, 2002.

[16] Mikhalev, A.Y. \& Oseledets, I.V., Adaptive nested cross approximation of non-local operators. arXiv preprint 1309.1773, 2013.

[17] Barbarosie, C. \& Toader, A.M., Shape and topology optimization for periodic problems. Part i: The shape and the topological derivative. Structural and Multidisciplinary Optimization, 40, pp. 381-391, 2010.

[18] Barbarosie, C. \& Toader, A.M., Shape and topology optimization for periodic problems. part ii: Optimization algorithm and numerical examples. Preprint $C M A F$, 017, pp. 1-14, 2008.

[19] Tyrtyshnikov, E.E., Mosaic-skeleton approximations. Calcolo, 33(1), pp. 4757, 1996.

[20] Hackbusch, W., Khoromskij, B. \& Sauter, S.A., On $\mathcal{H}^{2}$-matrices. Springer, 2000.

[21] Ying, L., Biros, G. \& Zorin, D., A kernel-independent adaptive fast multipole algorithm in two and three dimensions. J Comput Phys, 196(2), pp. 591-626, 2004.

[22] Ostanin, I., Zorin, D. \& Oseledets, I., Toward fast topological-shape optimization with boundary elements. preprint arXiv, (1503.02383), pp. 1-5, 2015. 